\title{
DESENVOLVIMENTO DE Diabrotica speciosa (Germar) (COLEOPTERA: CHRYSOMELIDAE) EM DIFERENTES HOSPEDEIROS
}

\section{DEVELOPMENT OF Diabrotica speciosa (Germar) (COLEOPTERA: CHRYSOMELIDAE) IN DIFFERENT HOST PLANTS}

\author{
Crébio José Ávila ${ }^{1}$ José Roberto Postali Parra ${ }^{2}$
}

RESUMO

Este trabalho teve como objetivo determinar a influência do hospedeiro, sobre o desenvolvimento de larvas, adultos e a reprodução de $\boldsymbol{D}$. speciosa. Larvas recém-eclodidas foram criadas em "seedlings" de feijoeiro, soja, milho e batata enraizada, utilizando-se, como substrato, vermiculita esterilizada e umedecida. Determinou-se a duração e viabilidade do período larva-adulto e o peso de insetos (machos e fêmeas) recémemergidos. Em outro experimento, foi avaliada a capacidade de postura de D. speciosa. Para isso, ofereceram-se aos adultosfolhas de feijoeiro, soja, milho e batata. A duração e a viabilidade do período larva-adulto, bem como o peso dos insetos (machos e fêmeas) recém-emergidos, foram significativamente influenciados pela espécie hospedeira utilizada como alimento na fase larval. $O$ maior período de desenvolvimento das fases imaturas (larva + pupa) foi verificado em tubérculo de batata (36,5 dias) e o menor, em "seedlings" de milho (25,1 dias). Os maiores valores de viabilidade foram observadas em batata $(84,1 \%)$ e milho $(75,9 \%)$, enquanto que em soja $(30,1 \%)$ e feijoeiro $(9,4 \%)$ foram bastante reduzidos, mostrando serem estes dois hospedeiros inadequados para o desenvolvimento imaturo (larva + pupal) de D. speciosa. Os insetos alimentados com folhas de batata e feijoeiro apresentaram capacidades de postura significativamente superiores àqueles mantidos em folhas de soja ou milho. Verificou-se que "seedlings" de milho e radicelas de batata são adequados, como alimento, para larvas de D. speciosa, enquanto folhas de batata e feijão são os mais adequados para os adultos.

Palavras-chave: insecta, vaquinha, planta hospedeira fecundidade.

\section{SUMMARY}

The aim of this work was to determine the influence of the host plant, as food, on the larval and adult phases of $\boldsymbol{D}$. speciosa. Larvae of this insect were reared on seedlings of bean, corn, soybean, as well as on potato tubers kept in wet vermiculite. The following biological parameters were evaluated: duration and viability of the larva-adult period and the weight of newly emerged insects (males and females). The fecundity of $\boldsymbol{D}$. speciosa was also evaluated offering to the adults leaves of bean, soybean, corn and potato. The host plant offered as food on the larval phase influenced significantly the weight of the insects (males and females) as well as the duration and viability of the larva-adult period. The development time of the immature phases (larva + pupa) on potato (36.5 days) was longer than that on corn (25.1 days). The viability values (larva + pupa) were higher on potato $(84.1 \%)$ and corn $(75.9 \%)$ while on soybean and bean the viability was low $(30.1 \%$ and $9.4 \%$, respectively), characterizing these plants as inadequate hosts for the larval development of the insect. Adults of D. speciosa fed on potato and bean leaves presented fecundity significantly superior to that on soybean or corn leaves. Therefore, corn seedlings and potato tubers are appropriate to larval development of $\boldsymbol{D}$. speciosa, while leaves of potato and bean are suitable for adults.

Key words: insecta, corn rootworm, host plant, survival, fecundity.

\section{INTRODUÇÃO}

Diabrotica speciosa (Germar, 1824) (Coleoptera: Chrysomelidae) ocorre em diversos

\footnotetext{
${ }^{1}$ Engenheiro Agrônomo, Doutor em Entomologia, Pesquisador III da Embrapa Agropecuária Oeste, CP 661, 79804-970, Dourados, MS E-mail: crebio@cpao.embrapa.br. Autor para correspondência.

${ }^{2}$ Escola Superior de Agricultura Luiz Queiroz, Universidade de São Paulo, Departamento Entomologia, Fitopatologia e Zoologia Agrícola, 13418-900, Piracicaba, SP. 
países da América do Sul (ARÉSTEGUI, 1976; KRYSAN, 1986; BERCELLINI \& MALACALZA, 1994). O adulto alimenta-se da parte aérea de plantas, enquanto a larva, de raízes e tubérculos (GASSEN, 1989). Estudos em laboratório relacionados às técnicas de criação e bioecologia de D. speciosa são registrados na literatura (HAJI, 1981; PECCHIONI, 1988; SILVA-WERNECK $\boldsymbol{e t}$ al., 1995; ÁVILA et al., 2000; MILANEZ \& PARRA, 2000). No entanto, pesquisas para determinar a influência de plantas hospedeiras no desenvolvimento da larva e adultos dessa espécie são inexistentes. Trabalhos dessa natureza poderão auxiliar no entendimento dos fatores que determinam a dinâmica populacional da praga em condições de campo, fornecendo subsídios para o aperfeiçoamento e desenvolvimento de táticas para o seu controle.

Este trabalho teve como objetivo determinar a influência de quatro plantas hospedeiras, como alimento, para larvas e adultos de D. speciosa.

\section{MATERIAL E MÉTODOS}

A pesquisa foi conduzida no Departamento de Entomologia, Fitopatologia e Zoologia Agrícola da Escola Superior de Agricultura "Luiz de Queiroz" (ESALQ), Piracicaba, SP, Brasil. Os experimentos foram conduzidos sob condições controladas de temperatura $\left(25 \pm 2^{\circ} \mathrm{C}\right)$, umidade relativa $(60 \pm 10 \%)$ e de fotoperíodo (fotofase de 14h).

\section{Influência do hospedeiro na fase larval}

Larvas recém-eclodidas de $\boldsymbol{D}$. speciosa foram criadas em "seedlings" de feijoeiro ('Carioca'), soja ('IAC 8') e de milho ('BR 201') e em tubérculos enraizados de batata ('Bintje'), mantidos em vermiculita esterilizada conforme metodologia descrita por ÁVILA et al. (2000). Utilizou-se a equivalência de um "seedling" (feijão, soja e milho) para cada larva "inoculada" ou um tubérculo de batata para cada 20 larvas no recipiente de criação. $\mathrm{O}$ experimento foi conduzido em delineamento inteiramente casualizado, sendo os tratamentos constituídos pelos quatro hospedeiros, com sete repetições (recipientes de criação). Para cada tratamento, determinou-se a duração e viabilidade do período larva-adulto e o peso de adultos (machos e fêmeas) recém-emergidos.

\section{Influência do hospedeiro na fase adulta}

A fecundidade de $\boldsymbol{D}$. specios $\boldsymbol{a}$ foi avaliada alimentando-se os adultos (machos e fêmeas) com folhas de feijão, soja, milho e batata. Para evitar o efeito do alimento pré-imaginal sobre a fase adulta, os insetos foram criados em um único hospedeiro ("seedlings" de milho). Adultos recém-emergidos foram sexados, colocando-se um casal por gaiola cônica de material acrílico transparente $(13,0 \mathrm{~cm}$ de altura por 8,5 e $6,0 \mathrm{~cm}$ de diâmetros de base e topo, respectivamente), que continha pequenas aberturas no topo, vedadas com tecido "voil" para permitir aeração. Os folíolos dos hospedeiros foram mantidos em pequenos vidros com água no interior da gaiola.

Um recipiente plástico $(3,0 \mathrm{~cm}$ de diâmetro por $1,5 \mathrm{~cm}$ de altura), que continha gaze umedecida de cor preta, foi utilizado como substrato para oviposição (MILANEZ, 1995). O alimento foi trocado a cada três dias, ocasião em que também efetuava-se a contagem de ovos no substrato. A mortalidade de adultos (machos e fêmeas), em cada tratamento, foi também registrada. As folhas dos quatro hospedeiros (feijoeiro, batata, soja e milho) com, aproximadamente, 15 dias de idade, foram analisadas quimicamente para determinação do teor de nitrogênio $(\mathrm{N})$, visando-se encontrar alguma relação entre o conteúdo desse nutriente na folha e a fecundidade do inseto. $\mathrm{O}$ teor de $\mathrm{N}$ foi determinado no Laboratório de Nutrição Mineral de Plantas do Departamento de Solos e Nutrição de Plantas da ESALQ/USP, baseando-se em SARRUGE \& HAAG (1974).

$\mathrm{O}$ ensaio foi conduzido em delineamento inteiramente casualizado, sendo os tratamentos representados pelas espécies hospedeiras, com 20 repetições (gaiola com um casal). As variáveis determinadas nos dois experimentos (fase larval e adulta) foram submetidas à análise de variância e as médias dos tratamentos comparadas pelo teste de Tukey, em nível de 5\% de erro.

\section{RESULTADOS E DISCUSSÃO}

A espécie hospedeira, utilizada como alimento na fase larval de D. speciosa, afetou a duração e a viabilidade do período larva-adulto, bem como o peso dos insetos produzidos. $\mathrm{O}$ maior período de desenvolvimento das fases imaturas (larva + pupa) foi verificado em tubérculo de batata (36,5 dias) e o menor, em "seedlings" de milho $(25,1$ dias), enquanto em soja e feijão foram observadas durações intermediárias (Tabela 1). Os valores de duração do período larva-adulto em milho foram similares aos encontrados por SILVA-WERNECK et al. (1995) e MILANEZ \& PARRA (2000), que utilizaram a mesma dieta. HAJI (1981) também criou D. speciosa em dieta mista, constituída por plântulas de milho e tubérculos enraizados de batatinha, obtendo duração média de 43,2 dias para o período larva-adulto, cerca de sete dias superior à obtida com batata, nesta pesquisa. 
Tabela 1 - Duração e viabilidade do período larva - adulta e peso de adultos de Diabrotica speciosa, por ocasião da emergência, criados em diferentes hospedeiros. Temperatura: $25 \pm 2^{\circ} \mathrm{C}$; UR: $60 \pm 10 \%$, fotofase: 14 horas.

\begin{tabular}{lcccc}
\hline \multirow{2}{*}{ Hospedeiro } & Duração & Viabilidade & \multicolumn{2}{c}{ Peso de adultos (mg) } \\
\cline { 2 - 5 } & (dias) & $(\%)$ & Macho & Fêmea \\
\hline Batata & $36,5 \pm 0,15 \mathrm{a}^{1}$ & $84,1 \pm 3,68 \mathrm{a}$ & $8,4 \pm 0,12 \mathrm{c}$ & $8,8 \pm 0,16 \mathrm{~b}$ \\
Milho & $25,1 \pm 0,23 \mathrm{c}$ & $75,9 \pm 1,50 \mathrm{a}$ & $9,5 \pm 0,19 \mathrm{~b}$ & $10,3 \pm 0,25 \mathrm{a}$ \\
Soja & $26,9 \pm 0,13 \mathrm{~b}$ & $30,1 \pm 0,84 \mathrm{~b}$ & $10,0 \pm 0,23 \mathrm{ab}$ & $10,4 \pm 0,17 \mathrm{a}$ \\
Feijão & $27,8 \pm 0,29 \mathrm{~b}$ & $9,4 \pm 2,17 \mathrm{c}$ & $10,6 \pm 0,25 \mathrm{a}$ & $10,0 \pm 0,24 \mathrm{a}$ \\
\hline
\end{tabular}

${ }^{1}$ Médias não seguidas da mesma letra, diferem estatisticamente entre si, pelo teste de Tukey, em nível de 5\% de erro.

As maiores viabilidades do período larvaadulto foram observadas em tubérculos de batata $(84,1 \%)$ e "seedlings" de nilho $(75,9 \%)$, as quais não diferiram entre si (Tabela 1). Esses percentuais de viabilidade são superiores aos obtidos por SILVA-WERNECK $\boldsymbol{e t}$ al. (1995) e MILANEZ \& PARRA (2000), que também utilizaram milho como hospedeiro larval, e por HAJI (1981) que utilizou radículas de milho + tubérculos de batatinha. Essas variações podem ser atribuídas às diferentes metodologias empregadas para criação do inseto, especialmente com relação ao substrato do sistema de criação, visto que, no presente trabalho, foi empregada vermiculita umedecida, enquanto nas outras técnicas, os autores utilizaram papel de filtro, solo e/ou areia para manutenção do alimento larval. A viabilidade do período larva-adulto foi muito reduzida em soja $(30,1 \%)$ e feijoeiro $(9,4 \%)$, caracterizando-os como hospedeiros inadequados em relação ao milho e à batata. No geral, independente do hospedeiro, houve uma relação inversa entre os valores de viabilidade e o peso de adultos recém-emergidos, especialmente com relação aos machos (Tabela 1).

A análise conjunta dos dados permite afirmar que o milho foi o hospedeiro mais adequado para criação de $\boldsymbol{D}$. speciosa, embora a viabilidade do período larva-adulto não tenha diferido estatisticamente daquela obtida em batata. Por outro lado, mesmo com baixa viabilidade, o inseto desenvolveu-se em "seedlings" de soja e feijão. Estes resultados indicam que, em condições de campo, na ausência de um hospedeiro preferencial, as larvas de $\boldsymbol{D}$. speciosa podem utilizar soja ou feijoeiro como hospedeiros alternativos para alimentação e reprodução. BRANSON \& KRYSAN (1981) também argumentaram que mesmo as espécies do gênero Diabrotica consideradas monófagas, ou com estreita gama de hospedeiros na fase larval, podem utilizar outros hospedeiros para completar seu ciclo de vida na ausência do hospedeiro preferencial e, consequientemente, garantir sua sobrevivência nos agroecossistemas. Já para adultos, os casais alimentados com folhas de batata e de feijoeiro apresentaram capacidade de postura superior àqueles mantidos em folhas de soja ou milho (Figura 1). Em milho, os insetos colocaram menos do que $2 \%$ dos ovos produzidos por aqueles alimentados em folhas de batata ou feijoeiro. A longevidade de adultos (machos e fêmeas) também foi afetada pelo tipo de alimento oferecido. Os insetos alimentados com folhas de milho apresentaram baixa sobrevivência durante o período de avaliação de postura, chegando a $100 \%$ de mortalidade aos 32 dias após o acasalamento, enquanto aqueles mantidos em folíolos de soja, feijoeiro e batata apresentaram mortalidades de 2,5, 13,2, e 22,5\%, respectivamente (Figura 2). Baseando-se nos valores de fecundidade e de sobrevivência de D. speciosa, nos quatro hospedeiros, pode-se afirmar que folhas de milho e de soja foram nutricionalmente menos adequadas para os adultos que folhas de batata ou de feijoeiro. SIEGFRIED \& MULLIN (1990) também constataram menor fecundidade de $\boldsymbol{D}$. virgifera virgifera, quando os adultos foram alimentados com

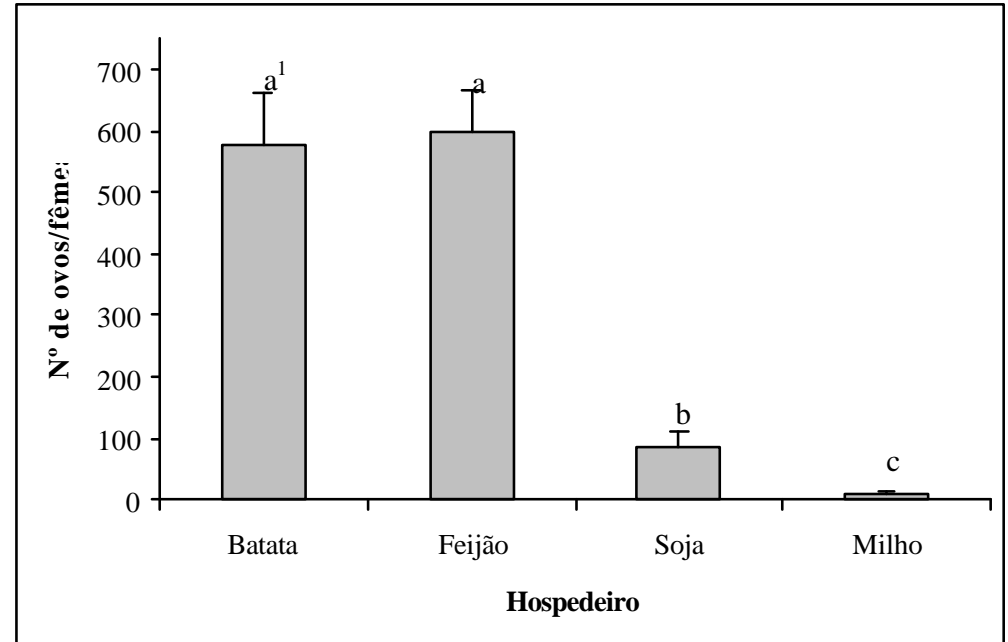

Figura 1 - Total de ovos produzidos por fêmea de Diabrotica speciosa (10 avaliações de postura), quando alimentados com diferentes hospedeiros na fase adulta. Temperatura: $25 \pm 2{ }^{\circ} \mathrm{C}$; UR: $60 \pm 10 \%$, fotofase: 14 horas. Letras diferentes sobre as barras indicam que os valores diferem estatisticamente entre si, pelo teste de Tukey, em nível de $5 \%$ de erro. 


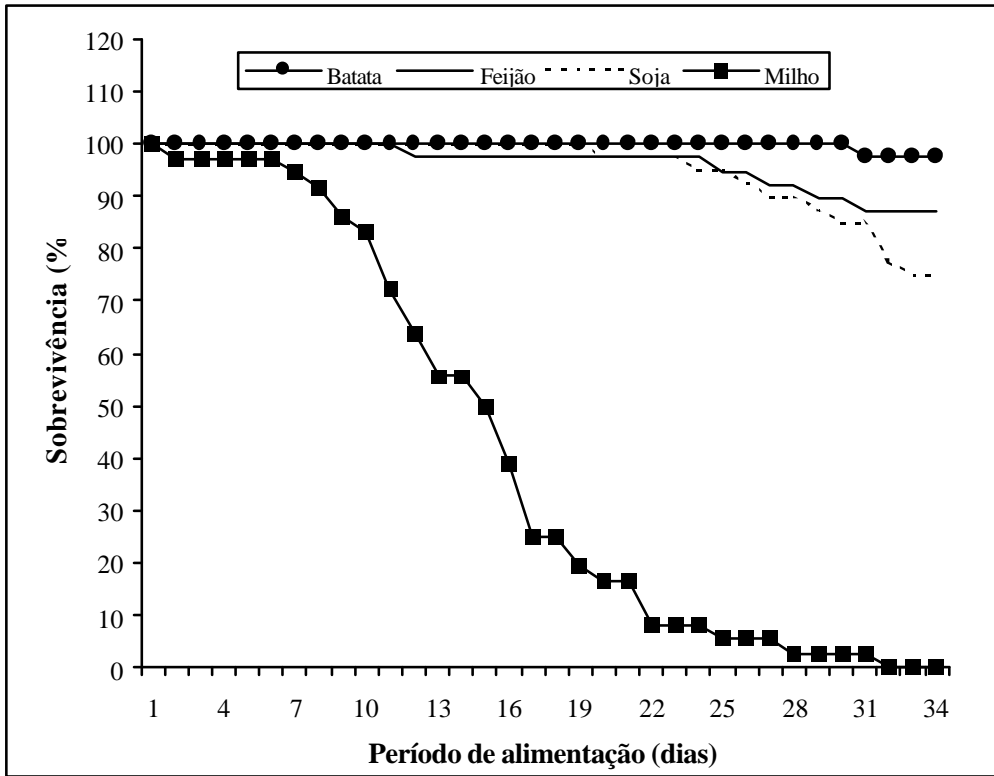

Figura 2 - Sobrevivência de adultos de Diabrotica speciosa nas gaiolas, quando alimentados com folhas dos diferentes hospedeiros. Temperatura: $25 \pm$ $2^{\circ} \mathrm{C}$; UR: $60 \pm 10 \%$, fotofase: 14 horas. espécies de insetos (AL-ZUBAIDI \& CAPINERA, 1984; WHEELER, 1996). No entanto, deve-se ressaltar que o nitrogênio foliar total, determinado quimicamente nas folhas dos hospedeiros, pode não estar todo disponível para ser utilizado pelos adultos de $\boldsymbol{D}$. speciosa e que sua concentração e disponibilidade no tecido foliar para a nutrição do inseto é uma característica de cada espécie de planta hospedeira (MATTSON, 1980).

Com base nos resultados obtidos, pode-se concluir que "seedlings" de milho e radicelas de batata constituíram os melhores alimentos para o desenvolvimento de larvas de $\boldsymbol{D}$. speciosa, enquanto folhas de batata e de feijão foram os mais adequados para os adultos.

folhas de milho, em relação àqueles mantidos em capítulos de girassol e flores de abóbora. Por outro lado, TENG et al. (1984) verificaram que adultos de $\boldsymbol{D}$. balteata alimentados com folíolos de batata apresentaram maior fecundidade que os mantidos em feijoeiro ou soja, diferindo, em parte, dos resultados encontrados no presente trabalho com D. speciosa. LANCE \& FISHER (1987) e NARANJO \& SAWYER (1987) também verificaram que a fecundidade e a longevidade de $\boldsymbol{D}$. barberi foram significativamente afetadas pelo tipo de dieta utilizada na fase adulta.

$$
\text { Segundo WHELEER }
$$

(1996), a capacidade de postura dos

insetos é determinada pela ovogênese, processo fisiológico que é regulado pela disponibilidade de nutrientes presentes no corpo da fêmea. Dessa forma, qualquer fator que afete a incorporação de nutrientes pelo inseto poderá afetar a ovogênese e, conseqüientemente, a taxa de postura. Através da análise foliar, verificou-se que a concentração de nitrogênio foi aparentemente menor em milho e maior na batata (Figura 3). A capacidade de postura de $\boldsymbol{D}$. speciosa pode estar relacionada às diferenças na quantidade do nitrogênio foliar encontradas nesses hospedeiros, como foi verificado para outras

\section{REFERÊNCIAS BIBLIOGRÁFICAS}

AL-ZUBAIDI, F.S., CAPINERA, J.L. Utilization of food and nitrogen by the beet armyworm, Spodoptera exigua (Hübner) (Lepidoptera: Noctuidae), in relation to food type and dietary nitrogen levels. Environmental Entomology, College Park, v.13, n.6, p.1604-1608, 1984.

ARÉSTEGUI, A.P. Plagas de la papa en Andahuaylas-Apurimac. Revista Peruana de Entomologia, Lima, v.19, n.1, p.97-98, 1976.

ÁVILA, C.J., TABAI, A.C.P., PARRA, J.R.P. Comparação de técnicas para criação de Diabrotica speciosa (Germar) (Coleoptera: Chrysomelidae) em dietas natural e artificial. Anais da Sociedade Entomológica do Brasil, Londrina, v. 29, n. 2, p. $257-267,2000$ 
BERCELLINI, N., MALACALZA, L. Plagas y predadores en soja en el noroeste de la provincia de Buenos Aires (Arg.). Turrialba, San Jose, v.44, n.4, p.244-254, 1994.

BRANSON, T.F., KRYSAN, J.L. Feeding and oviposition behavior and life cycle strategies of Diabrotica: an evolutionary view with implications for pest management. Environmental Entomology, College Park, v.10, n.6, p.826831,1981 .

GASSEN, D.N. Insetos subterrâneos prejudiciais às culturas no sul do Brasil. Passo Fundo : EMBRAPA-CNPT, 1989. 49p. (EMBRAPA-CNPT. Documentos, 13).

HAJI, N.F.P. Biologia, dano e controle do adulto de Diabrotica speciosa (Germar, 1824) (Coleoptera: Chrysomelidade) na cultura da batatinha (Solanum tuberosum L.). Piracicaba, 1981. 53p. Tese (Doutorado em Entomologia) - Escola Superior de Agricultura "Luiz de Queiroz", Universidade de São Paulo, 1981.

KRYSAN, J.L. Introduction: biology, distribution, and identification of pest Diabrotica. In: KRYSAN, J.L., MILLER, T.A. (Ed.) Methods for study of pest Diabrotica. New York : Springer Verlag, 1986. Cap.1, p.1-23.

LANCE, D.R., FISHER, J.R. Food quality of the various plant tissues for adults of the northern corn rootworm (Coleoptera: Chrysomelidae). Journal of the Kansas Entomological Society, Lawrence, v.60, n.3, p.462-466, 1987.

MATTSON, W.J. Herbivory in relation to plant nitrogen content. Annual Review of Ecology and Systematics, Palo Alto, v.11, p.119-161, 1980 .

MILANEZ, J.M., PARRA, J.R.P. Biologia e exigências térmicas de Diabrotica speciosa (Germar) (Coleoptera: Chrysomelidae) em laboratório. Anais da Sociedade Entomológica do Brasil, Londrina, v.29, n.1, p.23-29, 2000 .
MILANEZ, J.M. Técnicas de criação e bioecologia de Diabrotica speciosa (Germar, 1824) (Coleoptera: Chrysomelidae). Piracicaba, 1995. 102p. Tese (Doutorado em Entomologia) Escola Superior de Agricultura "Luiz de Queiroz", Universidade de São Paulo, 1995.

NARANJO, S.E., SAWYER, A.J. Reproductive biology and survival of Diabrotica barberi (Coleoptera: Chrysomelidae): effect of temperature, food, and seasonal time of emergence. Annals of the Entomological Society of America, College Park, v.80, n.6, p.841-848, 1987.

PECCHIONI, M.T.D. Crianza de Diabrotica speciosa (Coleoptera: Chrysomelidae) bajo condiciones de laboratorio. Revista Peruana de Entomologia, Lima, v.31, p.86-90, 1988.

SARRUGE, W, HAAG, H.P. Análise químicas em planta. Piracicaba : ESALQ-Departamento de Química, 1974. 56p.

SIEGFRIED, B.D., MULLIN, C.A. Effects of alternative host plants on longevity, oviposition, and emergence of western and northern corn rootworms (Coleoptera: Chrysomelidae). Environmental Entomology, Lanham, v.19, n.3, p.474-480, 1990.

SILVA-WERNECK, J.O., DE FARIA, M.R., ABREU NETO M.V., et al. Técnica de criação de Diabrotica speciosa (Germ.) (Coleoptera: Chrysomelidae) para bioensaios com bacilos e fungos entomopatogênicos. Anais da Sociedade Entomológica do Brasil, Piracicaba, v.24, n.1, p.45-52, 1995.

TENG, H.J., WADDILL, V., SLANSKY, F., et al. Performance and host preference of adult banded cucumber beetles, Diabrotica balteata when offered several crops. Journal of Agricultural Entomology, v.1, n.4, p.330-338, 1984.

WHEELER, D. The role of nourishment in oogenesis. Annual Review of Entomology, Palo Alto, v.41, p.407-431, 1996. 